\title{
ACTIVE VIBRATION CONTROL USING SELF-SENSING ACTUATORS : AN EXPERIMENTAL COMPARISON OF PIEZOELECTRIC AND ELECTROMAGNETIC TECHNOLOGIES
}

\author{
Romain Boulandet* \\ Groupe d'Acoustique de l'Université de Sherbrooke \\ Université de Sherbrooke \\ 2500 boul. de l'Université, J1K 2R1 \\ Sherbrooke, Québec, Canada \\ Email: romain.boulandet@usherbrooke.ca
}

\author{
Anik Pelletier, Philippe Micheau, Alain Berry \\ Groupe d'Acoustique de l'Université de Sherbrooke \\ Université de Sherbrooke \\ 2500 boul. de l'Université, J1K 2R1 \\ Sherbrooke, Québec, Canada
}

\begin{abstract}
The paper addresses the practical implementation of active vibration control using self-sensing actuators, intending to equip smart structures. The control objective is to reduce the structural vibration of a simply-supported plate subject to time-harmonic excitation. The key challenge is to use a self-sensing actuator instead of a sensor-actuator pair to reject the primary disturbance at the control point. In this study, two types of self-sensing actuators designed from a PZT patch and an electrodynamic inertial exciter are discussed, and their overall performance is compared in terms of reduction of flexural energy and power consumption. Both technologies have proven to be efficient in achieving a timeharmonic vibration control and may be used alternately, depending on the application at hand.
\end{abstract}

Keywords: Self-sensing actuator, Active vibration control, Piezoelectric actuator, Inertial exciter, Time-harmonic control

\section{INTRODUCTION}

An effective way to control vibration of mechanical structures is to use dynamic elements with properly chosen mechanical input impedance. The most common example is the shock absorber or vibration damper that is tuned to the resonance frequency of the host structure to be damped. Besides the passive approach, a wide variety of active control strategies can be implemented to achieve vibration reduction [1,2]. In most cases,

*Address all correspondence to this author. electromechanical transducers such as accelerometer, inertial exciter, PVDF- or PZT-type piezoelectric materials are needed for both the sensing and actuation $[3,4,5,6,7,8]$. The way a vibrating structure can be observed and controlled is a key aspect in the implementation of active control strategies. The stability of the controlled system, in particular, is highly dependent on the locations of the sensors and actuators. In feedback control systems, for example, it is advantageous to use a pair of collocated actuators-sensors. When dual variables are involved in both sensing and actuation, their product is proportional to the power supplied to the structure and the controlled system is positive real, provided the uncontrolled structure is dissipative $[9,10]$. For these reasons, it is worth considering the use of a self-sensing actuator (SSA) instead of a sensor-actuator pair.

A self-sensing actuator is basically a reversible electromechanical transducer, generally piezoelectric or electromagnetic, which uses simultaneously the dual function of sensing and actuation. The idea of self-sensing actuation was developed concurrently by Hagood et al. [11] and Dosch et al. [12] in the early nineties, where the PZT elements were used as sensors and actuators simultaneously to reduce implementation, cost and complexity, by achieving truly collocated control. The principle was later implemented to achieve vibration control on a cantilever beam [9], active structural acoustic control on a simply supported plate [13], vibration damping [14], to develop a sensorless technique for active noise control using an electrodynamic loudspeaker [15], or for biomedical application using an electro- 
magnetic actuator [16]. In addition to being dual, the control and observation variables are perfectly collocated, thereby ensuring that the controlled system remains passive.

In this article, we present and compare two ways to develop self-sensing actuators for time-harmonic vibration control. Selfsensing actuation is attractive for such applications because it can modify the local mechanical impedance of a structure in a predictable and desired manner without the need for additional sensors. The remaining is organized as follows. First, the coupled electromechanical model between the transducer and the host structure is described through an impedance-based approach, including the electromagnetic and piezoelectric coupling terms. Then, the baseline concept of the virtual mechanical impedance is presented, along with its practial implementation using the complex envelope controller. Experimental results performed on a simply-supported plate excited by a transverse force are given, wherein the overall performance is compared in terms of flexural energy reduction and transducer power consumption. Last, concluding remarks on the choice of technology according to the application to be implemented are provided.

\section{FLEXURAL STRUCTURE MODEL}

The system is composed of a flexible structure (an aluminum thin plate, as shown in Fig. 1), excited by a primary transverse force. A secondary source is then applied to control the vibration response. Under pure bending assumption, the velocity response $v_{s}(t)=\operatorname{Re}\left[\underline{v}_{s} \exp (j \omega t)\right]$ of the plate at a location $(x, y)$ can be expressed using the modal summation formulation [2] of the complex velocity, as

$$
\underline{v}_{s}(x, y)=j \omega \sum_{m=1}^{\infty} \sum_{n=1}^{\infty} \underline{q}_{m n} \phi_{m n}(x, y)
$$

where $\underline{q}_{m n}$ is the complex modal amplitude and $\phi_{m n}$ is the $m, n$ th mode shape function, which is defined for a simply supported $L_{x} \times L_{y}$ rectangular thin plate, as

$$
\phi_{m n}(x, y)=\sin \left(\gamma_{m} x\right) \sin \left(\gamma_{n} y\right)
$$

where $\gamma_{m}=m \pi / L_{x}$ and $\gamma_{n}=n \pi / L_{y}$.

Considering the general case of a time-harmonic excitation of the form $f(t)=\operatorname{Re}[\underline{F} \exp (j \omega t)]$, where $\underline{F}$ is the complex phasor of a transverse force, $\omega$ is the angular frequency and $j=\sqrt{-1}$, the complex modal amplitude $\underline{q}_{m n}$ can be written as

$$
\underline{q}_{m n}=\frac{1}{\mu L_{x} L_{y}} \frac{\underline{F}_{m n}}{\omega_{m n}^{2}-\omega^{2}}
$$

where $\underline{F}_{m n}$ is the corresponding modal force that depends on the nature of the excitation, $\mu$ is the mass per unit area (in $\mathrm{kg} \mathrm{m}^{-2}$ ),

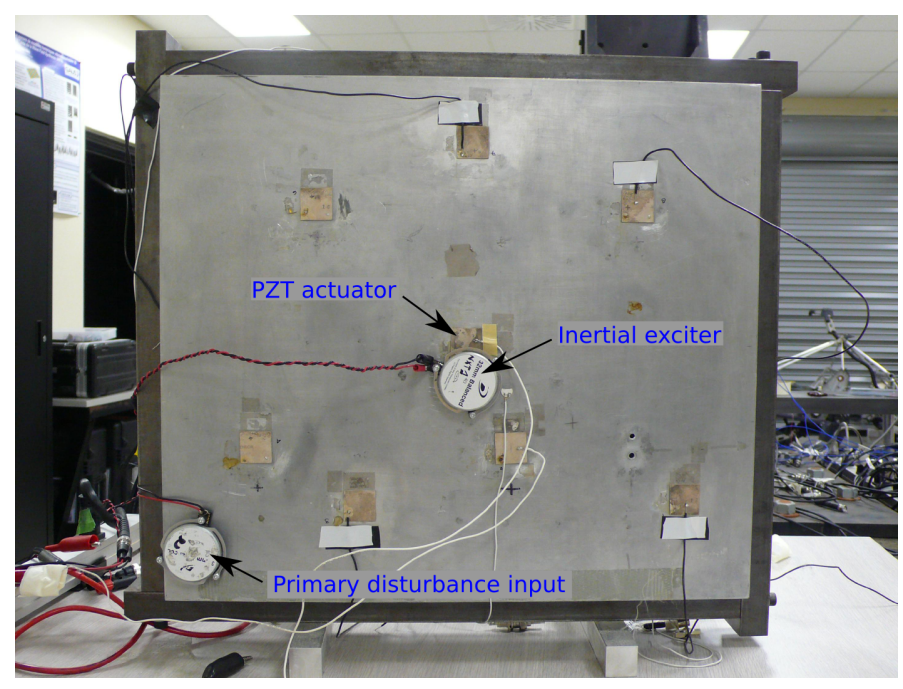

FIGURE 1: PICTURE OF THE EXPERIMENTAL SETUP.

and $\omega_{m n}$ is the natural frequency (in $\operatorname{rad~s}^{-1}$ ) of the $m, n$-th mode, given by

$$
\omega_{m n}=\sqrt{\frac{E h^{3}}{12\left(1-v^{2}\right) \mu}}\left(\gamma_{m}^{2}+\gamma_{n}^{2}\right)
$$

where $\underline{E}=E(1+j \eta)$ is the Young's modulus (in Pa) including the structural damping factor $\eta, h$ is the thickness (in $\mathrm{m}$ ) and $v$ is the Poisson's ratio of the plate. The physical parameters of the plate can be found in Table 1. Note that the fluid loading by the surrounding acoustic medium is neglected in the plate response.

TABLE 1: PHYSICAL PARAMETERS OF THE PLATE.

\begin{tabular}{lccc}
\hline Parameter & Notation & Value & Unit \\
\hline Width & $L_{x}$ & 0.481 & $\mathrm{~m}$ \\
Length & $L_{y}$ & 0.421 & $\mathrm{~m}$ \\
Thickness & $h$ & 3.18 & $\mathrm{~mm}$ \\
Young's modulus & $E$ & $68.510^{9}$ & $\mathrm{~Pa}$ \\
Mass density & $\rho$ & 2700 & $\mathrm{~kg} \mathrm{~m}^{-3}$ \\
Poisson ratio & $v$ & 0.33 & \\
Structural damping factor & $\eta$ & 0.005 & \\
\hline
\end{tabular}




\section{COUPLED ELECTRODYNAMIC ACTUATOR-PLATE MODEL}

An electrodynamic inertial exciter is a reversible voice coil transducer which has capability to sustain and propagate input vibrational energy. When attached to a host mechanical structure subject to vibration, the equation of motion of the inertial exciter derives from the Newton's second law and can be written as [17]

$$
j \omega M_{a} \underline{v}_{a}=B l \underline{i}-\left(R_{a}+K_{a} / j \omega\right)\left(\underline{v}_{a}-\underline{v}_{s}\right)
$$

where $\underline{v}_{a}$ and $\underline{v}_{s}$ are the velocities of the moving mass and host structure at the base of the exciter, $M_{a}, R_{a}, K_{a}$ are the mass, damping coefficient, and stiffness of the mount, respectively, and $B l \underline{i}$ is the force of electric origin resulting from the magnetic field acting on a moving free charge (current).

The governing equation of the electrical dynamics is based on Kirchhoff's circuit laws and can be written as

$$
\underline{u}=\left(R_{e}+j \omega L_{e}\right) \underline{i}+\underline{\varepsilon}
$$

where $\underline{u}$ is the input voltage applied to the transducer terminals, $i$ is the electrical current flowing through the coil, and $\underline{\varepsilon}=-B l\left(\underline{v}_{s}-\underline{v}_{a}\right)$ is the back electromotive force (voltage) induced in the coil during motion. The constant parameters $R_{e}$ and $L_{e}$ are the dc resistance and self inductance of the coil. The physical parameters of the inertial exciter used in this study can be found in Table 2 .

Upon actuation using an electrically-excited inertial actuator, for example, the transverse velocity response of the plate at the same location is assumed to be given by

$$
\underline{v}_{s}=-j \omega M_{a} \underline{Y} \underline{v}_{a}
$$

TABLE 2: PHYSICAL PARAMETERS OF THE INERTIAL EXCITER MEASURED IN SMALL-SIGNAL RANGE.

\begin{tabular}{lccc}
\hline Parameter & Notation & Value & Unit \\
\hline dc resistance & $R_{e}$ & 3.36 & $\Omega$ \\
Voice coil inductance & $L_{e}$ & 0.15 & $\mathrm{mH}$ \\
Force factor & $B l$ & 4.4 & $\mathrm{~N} \mathrm{~A}^{-1}$ \\
Moving mass & $M_{a}$ & 0.105 & $\mathrm{~kg}$ \\
Damping coefficient & $R_{a}$ & 0.31 & $\mathrm{~N} \mathrm{~m}^{-1} \mathrm{~s}$ \\
Suspension stiffness & $K_{a}$ & $14.210^{3}$ & $\mathrm{~N} \mathrm{~m}^{-1}$ \\
Resonance frequency & $f_{c}$ & 59.6 & $\mathrm{~Hz}$ \\
\hline
\end{tabular}
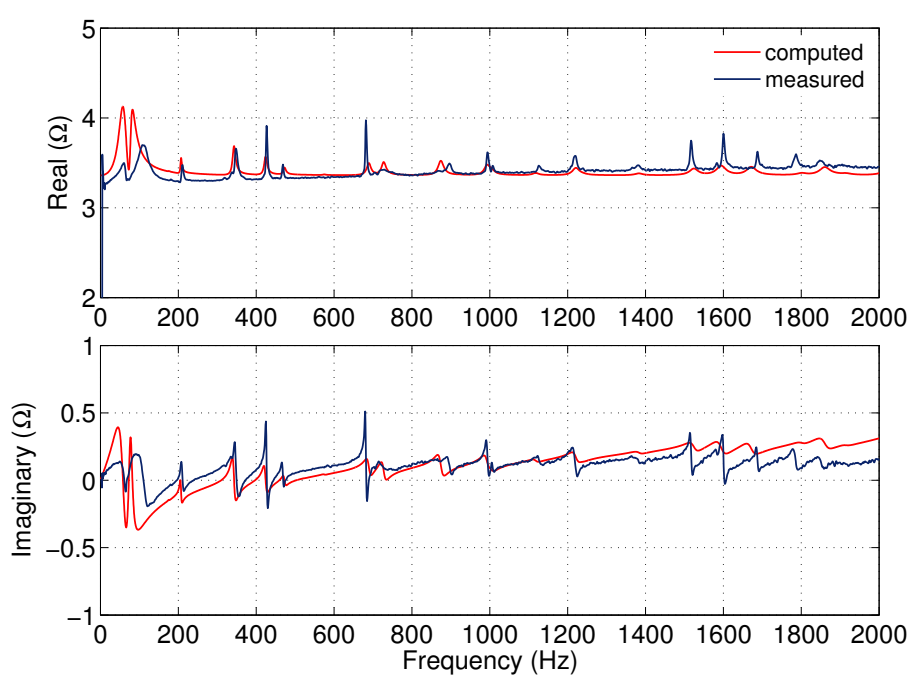

FIGURE 2: INPUT ELECTRICAL IMPEDANCE OF THE INERTIAL EXCITER WHEN COUPLED TO THE PLATE.

where $-j \omega M_{a} \underline{v}_{a}$ is the inertia force due to the vibration of the mass, and $\underline{Y}$ is the mobility function of the plate (in $\mathrm{m} \mathrm{s}^{-1} \mathrm{~N}^{-1}$ ) which can be derived from Eqs. (1) to (4), as

$$
\underline{Y}=-j \omega \frac{1}{\mu L_{x} L_{y}} \sum_{m=1}^{\infty} \sum_{n=1}^{\infty} \frac{\phi_{m n}^{2}(x, y)}{\omega_{m n}^{2}-\omega^{2}}
$$

Substituting Eq. (7) in Eqs. (5) and (6), the input electrical impedance (in $\Omega$ ) of the inertial exciter attached to the plate is given by

$$
\frac{\underline{u}}{\underline{i}}=\underline{Z}_{e}+\frac{(B l)^{2}}{\underline{Z}_{m a}-\frac{\left(j \omega M_{a}\right)^{2} \underline{Y}}{1+j \omega M_{a} \underline{Y}}}
$$

where $\underline{Z}_{e}=R_{e}+j \omega L_{e}$ is the blocked electrical impedance and $\underline{Z}_{m a}=j \omega M_{a}+R_{a}+K_{a} / j \omega$ is the mechanical impedance of the voice coil actuator. Figure 2 illustrates the frequency response function of the input electrical impedance of the inertial exciter at the control location. As shown in Fig. 2, the first natural frequencies of the plate can be clearly seen and the transducer's natural resonance strongly interacts with the first structural resonance at $75 \mathrm{~Hz}$.

Substituting now Eq. (7) in Eqs. (5) and (6) and after some further manipulations, the plate velocity can also be expressed as

$$
\underline{v}_{s}=-\frac{\underline{Z}_{m a}}{j \omega M_{a} B l}\left(\underline{u}-\underline{Z}_{e} \underline{i}\right)+\frac{B l}{j \omega M_{a}} \underline{i}
$$


and assuming a time-harmonic excitation such that $\sqrt{K_{a} / M_{a}}<$ $\omega<R_{e} / L_{e}$, i.e. above the natural resonance of the inertial exciter and below the cut-off frequency of the coil electrical filter, a simplified expression of Eq. (10) can be obtained as

$$
\underline{v}_{s} \simeq-\frac{\underline{u}}{B l}+\left(\frac{R_{e}}{B l}-j \frac{B l}{\omega M_{a}}\right) \underline{i}
$$

Equation (11) is the basis of the self-sensing electrodynamic actuator and will be used as an estimate of the structure velocity at the base of the inertial exciter in the following.

\section{COUPLED PIEZOELECTRIC ACTUATOR-PLATE MODEL}

Piezoceramic materials, such as lead zirconate titanate (PZT) patches for example, are reversible electromechanical transducers that can be used for both actuation and sensing when attached to a host structure. A PZT actuator deforms proportionally to the electric field applied to it with a direction determined by the piezoelectric charge constants (inverse piezoelectric effect). Conversely, when the piezoelectric material is subject to mechanical strain, electric charges, proportional to the strain and depending on the piezoelectric stress constant, are generated in the material (direct piezoelectric effect) [18]. When bonded to a flexural structure and driven by a voltage, for example, a bending moment can be created on the structure through the in-plane expansion and contraction of the PZT, causing the structure to vibrate $[3,4]$. Like the inertial exciter, the piezoelectric self-sensing actuator will seek to combine the dual function of sensing and actuation. However, the output current is partly due to the capacitive effect of the piezoelectric material and to the mechanical deformation of the structure. Unlike an inertial exciter, the PZT actuator creates strong local bending when actuated, which does not contribute to the global vibration of the plate [19]. When intended to be used as a self-sensing actuator, the output current of the PZT actuator needs to be corrected so as to minimize the contribution of the effects mentioned above. The processing needed for extracting the current produced by the bending moment of the structure is a model-based approach [20] and is implemented digitally. More details on the correction method can be found in [21].

The total current output of a PZT patch coupled to an elastic structure is the sum of two components: $\underline{i}_{c}$ the functional electrical current and $\underline{i}_{m}$ the current generated by strain effect on the PZT due to the coupled structure. It can be written as

$$
\underline{i}=\underline{i}_{c}+\underline{i}_{m}=\frac{\underline{u}}{\underline{Z}_{e}}+\underline{i}_{m}
$$

where $\underline{u}$ is the voltage applied at the PZT input terminals and $\underline{Z}_{e}$ is the electrical impedance of the piezoceramic element [22], as

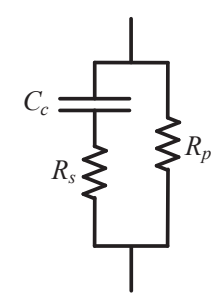

FIGURE 3: EQUIVALENT CIRCUIT OF THE PIEZOELECTRIC ELEMENT.

shown in Fig. 3. The expression of $\underline{Z}_{e}$ is

$$
\underline{Z}_{e}=\frac{1+j \omega C_{c} R_{s}}{\frac{1}{R_{p}}+j \omega C_{c}\left(1+\frac{R_{s}}{R_{p}}\right)}
$$

where $C_{c}$ is the measured capacitance and the resistances $R_{s}$ and $R_{p}$ are not known a priori but can be determined experimentally.

PZT actuators are often used in a symmetric configuration with two patches located at the same position but on each side of the plate. However, with self-sensing actuation, an asymmetric configuration (a single actuator on one side of the plate) is preferred. In that case, assuming that the plate and the actuator are of infinite dimension and the adhesive layer thickness is almost zero, the bending moment applied by the actuator onto the plate may be calculated $[23,24]$ and upon actuation using a voltage source $\underline{u}$, the complex modal amplitude (3) can be written as

$$
\underline{q}_{m n}=-\frac{4}{\mu L_{x} L_{y}} \frac{\underline{E}}{1-v} \frac{c h^{3}}{12} \frac{\gamma_{m}{ }^{2}+\gamma_{n}^{2}}{\gamma_{m} \gamma_{n}} \frac{\Delta \cos _{a}}{\omega_{m n}{ }^{2}-\omega^{2}} \frac{d_{31}}{h_{a}} \underline{u}
$$

where

$$
\begin{aligned}
c & =\frac{24 K \rho_{a}\left(2+\rho_{a}\right)}{h\left(16+32 K \rho_{a}+24 K \rho_{a}^{2}+8 K \rho_{a}^{3}+K^{2} \rho_{a}{ }^{4}\right)} \\
K & =\frac{E_{a}}{1-v_{a}} \frac{1-v}{\underline{E}} \\
\rho_{a} & =2 \frac{h_{a}}{h}
\end{aligned}
$$

and where $h_{a}$ is the thickness of the PZT patch, $d_{31}$ is the charge piezoelectric constant, $\underline{E}=E(1+j \eta)$ and $\Delta \cos _{a}=\left[\cos \left(\gamma_{m} x_{1}\right)-\cos \left(\gamma_{m} x_{2}\right)\right]\left[\cos \left(\gamma_{n} y_{1}\right)-\cos \left(\gamma_{n} y_{2}\right)\right] . x_{1}$, $x_{2}, y_{1}$ and $y_{2}$ are the physical limits of the PZT. The physical parameters of the piezoceramic material can be found in Table 3 . 
After some further manipulations, $\underline{i}_{m}$ may be written as

$$
\begin{aligned}
\underline{i}_{m}=j \omega \frac{4}{\mu L_{x} L_{y}} & \frac{\underline{E}}{1-v} \frac{c h^{3}}{12} \frac{h+h_{a}}{2} e_{31} \\
& \sum_{m=1}^{\infty} \sum_{n=1}^{\infty}\left(\frac{\gamma_{m}^{2}+\gamma_{n}^{2}}{\gamma_{m} \gamma_{n}}\right)^{2} \frac{\Delta \cos _{a}^{2}}{\omega_{m n}{ }^{2}-\omega^{2}} \frac{d_{31}}{h_{a}} \underline{u}
\end{aligned}
$$

As presented in [19], the contribution to the mechanical current $\underline{i}_{m}$ of the higher frequency modes $\left(\omega_{m n}{ }^{2} \gg \omega^{2}\right)$ may be expressed as a stiffness $K_{n f}$ and involves a local vibration response of the plate around the PZT transducer. Conversely, denoting the contribution $\underline{i}_{b}$ of the total current which is due to lower order modes and thus contains the global vibration of the plate, Eq. (18) may be rewritten as

$$
\underline{i}_{m}=\frac{j \omega}{K_{n f}} \underline{u}+\underline{i}_{b}
$$

Note that $\underline{i}_{b}$ is the variable to be considered in the context of active control of global vibrations.

Substituting now Eq. (19) into Eq. (12), the current due to the global vibration may be obtained from the total current and voltage at the PZT terminals, as

$$
\underline{i}_{b}=\underline{i}-\left(\frac{1}{\underline{Z}_{e}}+\frac{j \omega}{K_{n f}}\right) \underline{u}=\underline{i}-\underline{G} \underline{u}
$$

which has been experimentally verified to be proportional to the transverse velocity of the structure $\underline{v}_{s}$ for a given frequency. The

TABLE 3: PHYSICAL PARAMETERS OF THE PZT ACTUATOR.

\begin{tabular}{lccc}
\hline Parameter & Notation & Value & Unit \\
\hline Width & $l_{x}$ & 25.4 & $\mathrm{~mm}$ \\
Length & $l_{y}$ & 25.4 & $\mathrm{~mm}$ \\
Thickness & $h_{a}$ & 1.02 & $\mathrm{~mm}$ \\
Young's modulus & $E_{a}$ & 64.5 & $\mathrm{GPa}$ \\
Poisson ratio & $v_{a}$ & 0.32 & \\
Piezoelectric constant & $d_{31}$ & $-17510^{-12}$ & $\mathrm{C} \mathrm{N}^{-1}$ \\
Permitivity & $\varepsilon_{33}^{\sigma}$ & 1750 & \\
Capacitance & $C_{c}$ & 7.19 & $\mathrm{nF}$ \\
\hline
\end{tabular}
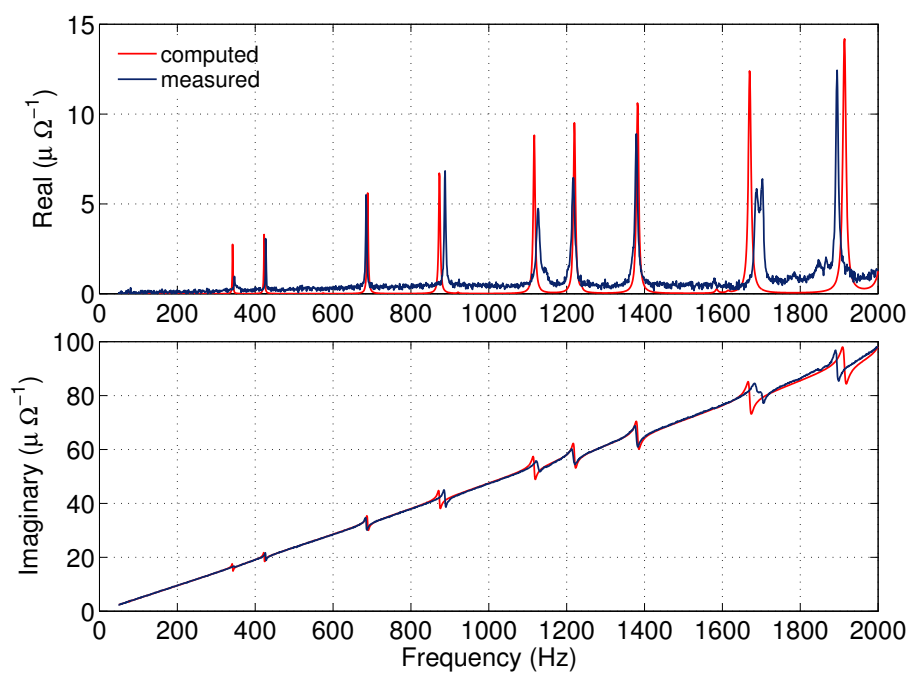

FIGURE 4: INPUT ELECTRICAL ADMITTANCE OF THE PIEZOELECTRIC ACTUATOR WHEN COUPLED TO THE PLATE.

closed form expression of the compensation term $\underline{G}$ may be derived by combining Eqs. (13) and (20), as

$$
\underline{G}=\frac{1+\omega^{2} C_{c}{ }^{2} R_{s}\left(R_{s}+R_{p}\right)}{R_{p}\left(1+\omega^{2} C_{c}{ }^{2} R_{s}{ }^{2}\right)}+j \omega\left(\frac{1}{K_{n f}}+\frac{C_{c}}{1+\omega^{2} C_{c}{ }^{2} R_{s}{ }^{2}}\right)
$$

It has been shown in a previous work [21] that $\underline{G}$ can be approximated quite accurately over a limited frequency range by

$$
\underline{G} \simeq \kappa_{r} \omega^{2}+j \kappa_{i} \omega
$$

where $\kappa_{r}=C_{c}^{2} R_{S}\left(1+R_{S} / R_{p}\right)$ and $\kappa_{i}=C_{c}+1 / K_{n f}$ are constants which are determined experimentally with no primary excitation using a curve fitting method.

Figure 4 illustrates the measured and computed frequency response function of the input electrical admittance of the piezoelectric actuator near the center of the plate, in the absence of primary disturbance. In the case of the PZT actuator, the admittance is used instead of the impedance since the output of the transducer is a current and not a voltage as for the inertial exciter. Figures 2 and 4 thus show the ratio of the input signal and the transducer output signal in both cases. Similar to the inertial exciter, the first natural frequencies of the plate can be clearly seen from the measured electrical admittance over a frequency range of $0-2 \mathrm{kHz}$. In practice, $\kappa_{i}$ is the slope of the imaginary part of the admittance (Fig. 4) and includes both the capacitive behavior of the PZT and the local bending effect of the self-sensing actuator and $\kappa_{r}$ is the second order coefficient of the real part of the admittance. 
Then, upon self-sensing actuation, a simplified expression of Eq. (20) can be derived as

$$
\underline{i}_{b}=\underline{i}-\left(\kappa_{r} \omega^{2}+j \kappa_{i} \omega\right) \underline{u}
$$

Equation (23) is the basis of the self-sensing piezoelectric actuator that is implemented in the real-time controller.

\section{TIME-HARMONIC CONTROL}

The control objective is to reduce the structural vibration of a simply-supported plate which is subject to a time-harmonic excitation. The key challenge is to use a self-sensing actuator instead of a sensor-actuator pair to reject the primary disturbance at the control point. Figure 5 illustrates the block diagram of the control scheme in which $d$ refers to the disturbance. As can be seen in Fig. 5, the flow variable (controlled signal) $f$ depends on both the transducer output $y$ and the effort variable (control input) $\underline{e}$.

The time-harmonic disturbance rejection is implemented using a complex envelope controller, the function of which can be expressed as [25]

$$
\frac{\mathrm{d} \underline{x}}{\mathrm{~d} t}=-\mu \underline{C} \underline{f}
$$

where $\underline{C}=\underline{H}_{s}^{-1}$ and $\underline{H}_{s}$ is the system transfer function between the input $\underline{x}$ and the flow variable $f$, and $\mu$ is a gain coefficient. Note that Eq. (24) is shown to be equivalent to the implementation of a resonant controller [25]. Table 4 compares the expressions for the effort, flow, output variables and compensation which are required to obtain the flow variable from the effort input and transducer output, in accordance with Eqs. (11) and (20).

TABLE 4: EFFORT, FLOW, OUTPUT AND COMPENSATION.

\begin{tabular}{lccccc}
\hline Case & flow & effort & output & \multicolumn{2}{c}{ Compensation } \\
& $\underline{f}$ & $\underline{e}$ & $y$ & $\underline{\mathrm{G}}$ & $\underline{\mathrm{K}}$ \\
\hline $\begin{array}{l}\text { Inertial } \\
\text { exciter }\end{array}$ & $\underline{v}_{s}$ & $\underline{i}$ & $u$ & $R_{e}-j \frac{(B l)^{2}}{\omega M_{a}}$ & $-\frac{1}{B l}$ \\
$\begin{array}{l}\text { PZT } \\
\text { patch }\end{array}$ & $\underline{i}_{b}$ & $\underline{u}$ & $i$ & $\kappa_{r} \omega^{2}+j \kappa_{i} \omega$ & 1 \\
\hline
\end{tabular}

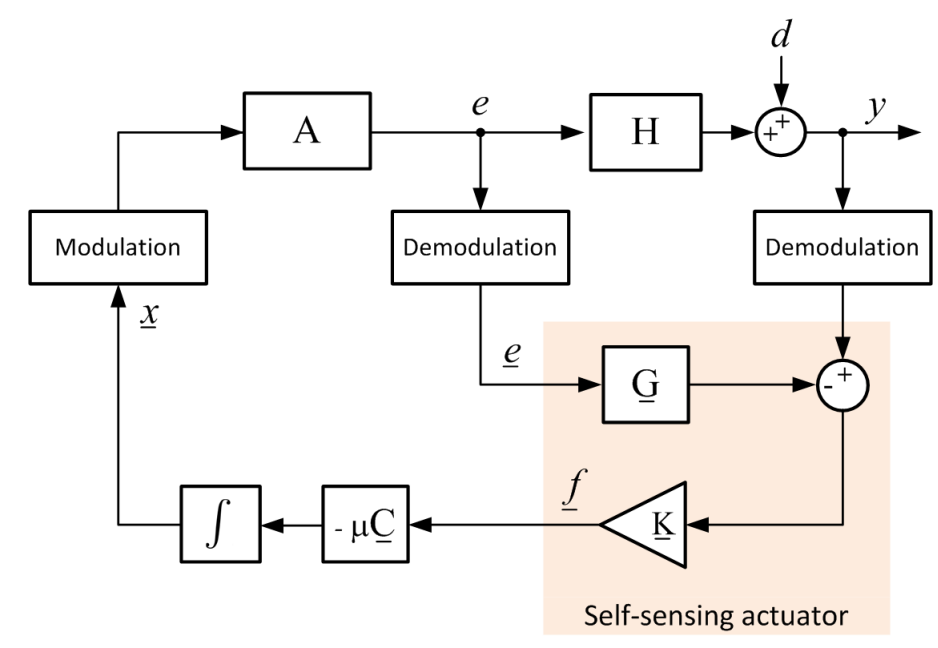

FIGURE 5: BLOCK DIAGRAM OF THE CONTROL SCHEME, INCLUDING THE COMPLEX ENVELOPE CONTROLLER.

The practical implementation of the control scheme given in Fig. 5 is done via a two-step process:

1. identification of the coupled structure-actuator system (offline), i.e. the parameters $\underline{G}, \underline{K}$ and $\underline{H}_{s}$,

2. real time self-sensing actuation in accordance with Eqs (11) and (23).

As shown in Fig. 5, $H$ is used to represent the system transfer function and $A$ is the electronic circuit transfer function between the input $\underline{x}$ and the effort variable $\underline{e}$. The complex envelope method is a complex-valued function of time (phasor) that is widely used in telecommunication to address different types of modulation. Modulation is used so that the frequencies in the baseband input signal are heterodyned up to the modulated carrier wave. Demodulation is then used to extract the original information-bearing signal from the modulated carrier wave. More details about analysis and synthesis of the complex envelope controller can be found in [26].

\section{RESULTS Experimental setup}

The experimental setup is illustrated in Fig. 1, where a 1/8 in. thick aluminium plate, with an area of $421 \mathrm{~mm} \times 481 \mathrm{~mm}$, is mounted on a rigid frame with simply-supported boundary conditions. The primary excitation is a time-harmonic transverse force generated by an electrically-excited inertial actuator which is similar to that used for self-sensing actuation. It is located near the corner of the plate, as shown in Fig. 1. The piezoelectric self-sensing actuator is bonded to the center of the plate ; the one designed from an electrodynamic transducer is attached nearby. 
Their location is chosen so that they can couple to the structural modes $(1,3)$ at $427 \mathrm{~Hz}$ and $(3,1)$ at $346 \mathrm{~Hz}$, i.e. away from the nodal lines of the structural modes to be controlled [8].

A VibroMet 500V Doppler laser vibrometer is also used to observe the vibration response of the plate near the location of the self-sensing actuators. Signal acquisition is done with 18 bit precision analog inputs at a sampling frequency of $20 \mathrm{kHz}$. The digital signal processing is performed using a Speedgoat performance real-time target machine running on Simulink ${ }^{\circledR}$ RealTime.

\section{Flexural kinetic energy} be [5]

The total flexural kinetic energy of the panel is defined to

$$
\underline{E}=\frac{\mu}{4} \int_{0}^{L_{x}} \int_{0}^{L_{y}}\left|\underline{v}_{s}(x, y)\right|^{2} d x d y
$$

where $\mu$ is the mass per unit area of the plate.

In practice, the vibrational velocity of the plate is measured on a grid of $11 \times 9$ scan points by scanning laser Doppler velocimetry. The total flexural kinetic energy is calculated as the sum of the squared velocities of each surface element.

Table 5 summarizes the measured control performance in case of time-harmonic disturance rejection at the structural modes $(1,3)$ and $(3,1)$ at $346 \mathrm{~Hz}$ at $427 \mathrm{~Hz}$, respectively, which are efficient acoustic radiators. As can be seen in Table 5, significant reduction of the total kinetic energy of the plate can be achieved with the two types of self-sensing actuators. The performances obtained after control are comparable for both cases. Part of the slight differences is likely due to the location of the two self-sensing actuators which are not exactly at the same place, as illustrated in Fig. 1.

\section{Power consumption}

The power consumption of the electromechanical transducers can be calculated from the phasor of the electrical variables, as

$$
P=\operatorname{Re}\left[\underline{u}^{*} \underline{i}\right]
$$

Table 5 summarizes the power consumption of the two types of self-sensing actuators when they are controlling the structural modes $(1,3)$ at $427 \mathrm{~Hz}$ and $(3,1)$ at $346 \mathrm{~Hz}$. As can be seen in Table 5, power consumption is much lower in the case of the piezoceramic actuator. It is most likely due to the capacitive nature of the piezoelectric materials and the phase shift between electrical variables which ensues. Further experiments are
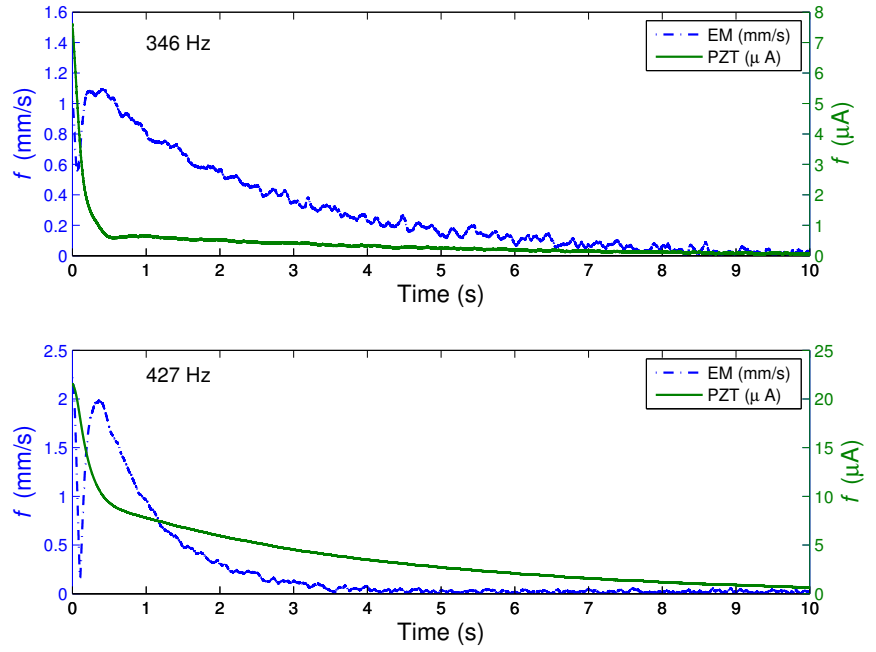

FIGURE 6: CONVERGENCE TIME TO ACHIEVE THE TIMEHARMONIC DISTURBANCE REJECTION.

planned to examine the cause of the difference.

Figure 6 shows the measured convergence time to achieve the time-harmonic disturbance rejection. As can be seen in Fig. 6, the two types of self-sensing actuators lead to effective rejection of the time-harmonic disturbance but with slightly different convergence times. Note that the measurements were performed for the same input voltage applied to the electricallyexcited inertial actuator used to provide the primary transverse force.

Figure 7 shows the normal velocity response of the plate subject to a time-harmonic transverse force excitation at $346 \mathrm{~Hz}$ and $427 \mathrm{~Hz}$, measured by scanning laser Doppler velocimetry. As can be seen in Fig. 7, the distribution of vibrational velocity on the plate is rather similar after control for both types of technologies. Note that the electromagnetic self-sensing actuator was removed for measurement without control.

\section{Discussion}

Experimental results clearly show that time-harmonic disturbance rejection can be achieved using the two types of transducers. As can be seen in Eqs. (11) and (20), the flow variable that is needed to achieve piezoelectric and electromagnetic self-sensing actuation is obtained using a similar compensation in both cases. Independently of the technology, the flow variable is clearly shown to vary proportionally with the true structural velocity, as illustrated in Fig. 8. This results in a generic controller architecture, as given in Fig. 5. However, there are differences that should be discussed. As illustrated in Fig. 8, the passive effect of the electrodynamic sensoriactuator upon the 
TABLE 5: CONTROL PERFORMANCE MEASURED AT 346 Hz AND 427 Hz ON THE SIMPLY-SUPPORTED PLATE.

\begin{tabular}{lcccc}
\hline & \multicolumn{2}{c}{ Mode $(3,1)$ at $346 \mathrm{~Hz}$} & \multicolumn{2}{c}{ Mode $(1,3)$ at $427 \mathrm{~Hz}$} \\
\cline { 2 - 5 } Case & Flexural energy & Power consumption & Flexural energy & Power consumption \\
\hline no control & $1.27410^{-9} \mathrm{~J}$ & - & $16.0910^{-9} \mathrm{~J}$ & - \\
inertial exciter & $0.04610^{-9} \mathrm{~J}$ & $0.32 \mathrm{~mW}$ & $0.20710^{-9} \mathrm{~J}$ & $0.47 \mathrm{~mW}$ \\
PZT actuator & $0.13610^{-9} \mathrm{~J}$ & $0.046 \mathrm{~mW}$ & $0.15710^{-9} \mathrm{~J}$ & $0.047 \mathrm{~mW}$ \\
\hline
\end{tabular}
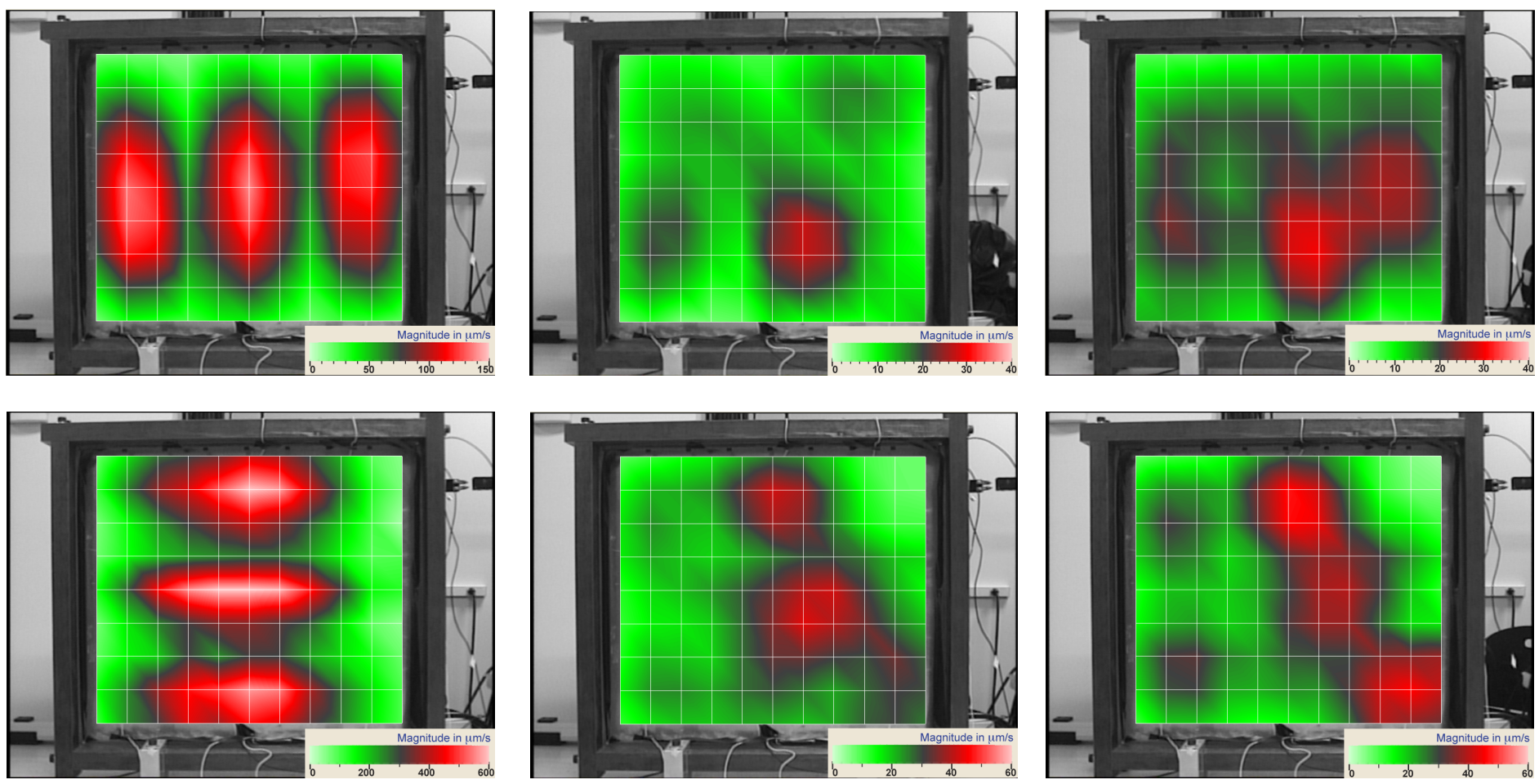

FIGURE 7: VIBRATIONAL VELOCITY MEASURED ON THE PLATE AT 346 Hz (TOP) AND 427 Hz (BOTTOM) WITH NO CONTROL (LEFT) AND WHEN USING THE ELECTROMAGNETIC (MIDDLE) AND PIEZOELECTRIC (RIGHT) SELF-SENSING ACTUATOR.

structure is greater. This explains the differences between the amplitudes of the measured signals before activating the control in both cases. Unlike the piezoceramic self-sensing actuator, the passive effect of the electrodynamic inertial exciter has a non negligible influence on the vibrational response of the plate. Nonetheless, an effective electromechanical coupling is ensured in most types of structure since the force is transversally applied, whereas the PZT patch must be matched to the host structure to achieve an efficient bending moment [24]. Properly chosen PZT patches can then be easily fitted to a structure without modifying significantly the the modes shape, as can an inertial exciter. Even though the power consumption is lower in the case of the piezo- electric actuator, currents generated can be very small and difficult to measure, particularly for flexural structures with a high inherent structural damping. Also shown in Fig. 8, for some applications it may be preferable to use the electrodynamic technology since the amplitude of the electrical quantities involved in the self-sensing actuation are easier to handle. The two technologies are therefore complementary and can be used alternatively, depending on the application. 

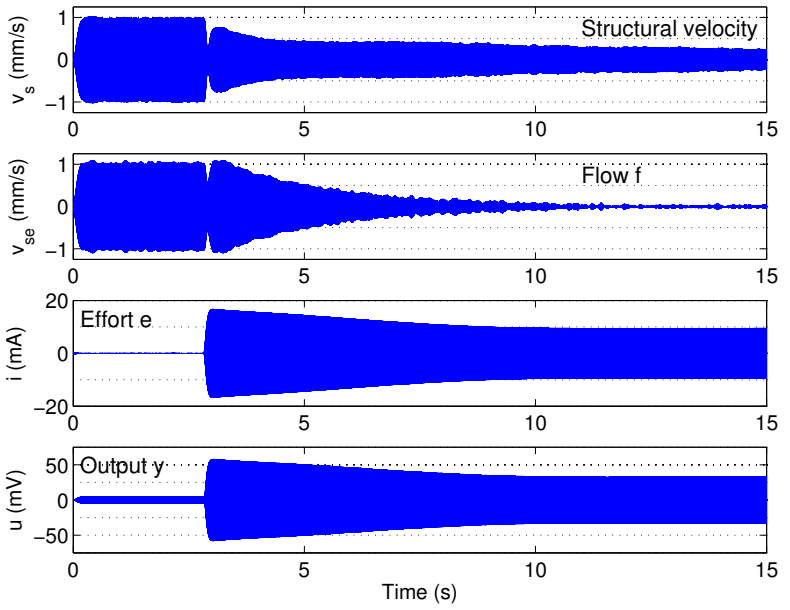

(a)
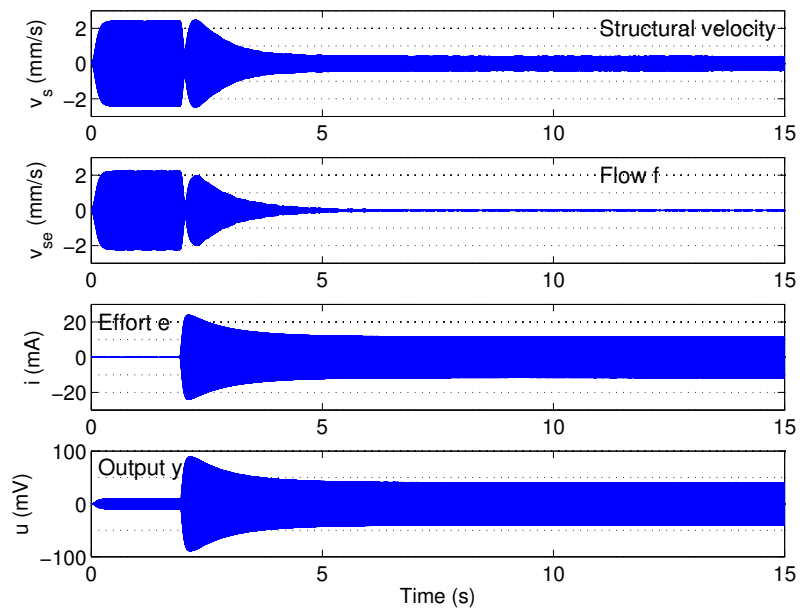

(c)
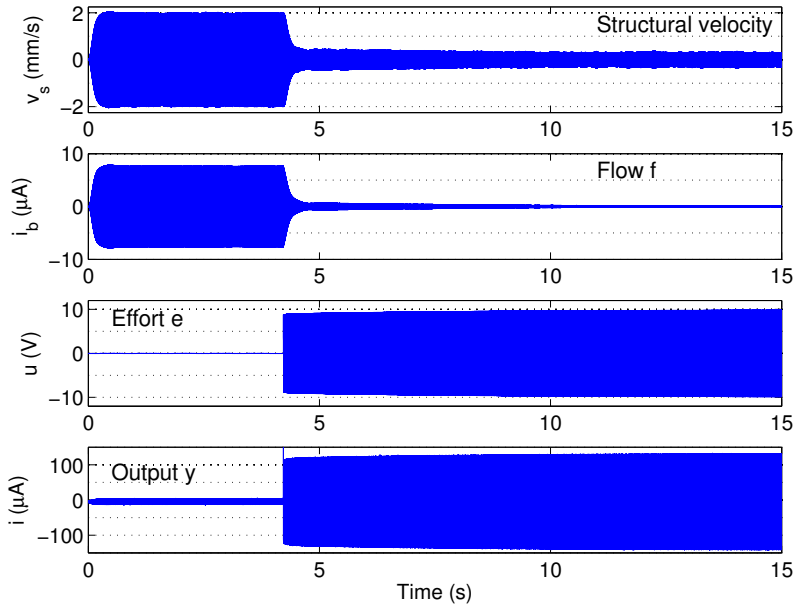

(b)
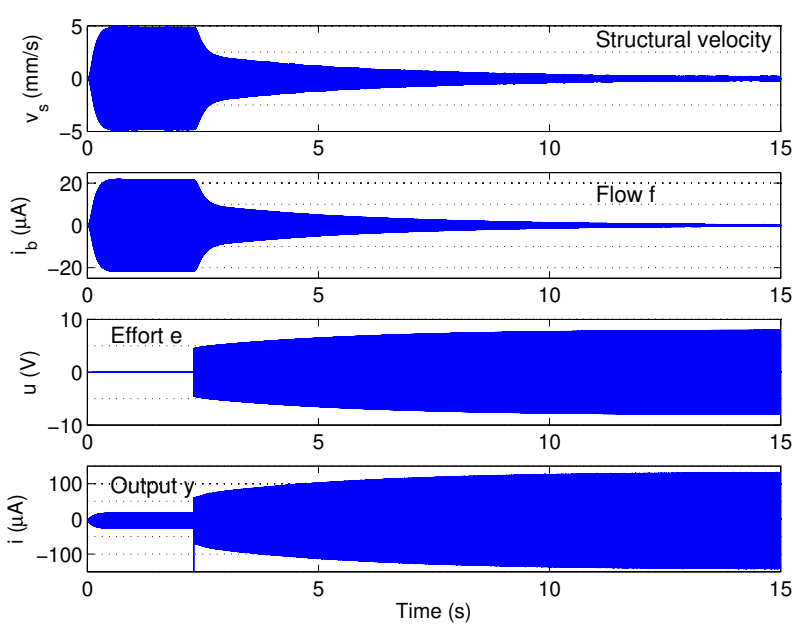

(d)

FIGURE 8: WAVEFORMS OF THE SIGNALS MEASURED WITH ELECTROMAGNETIC (LEFT) AND PIEZOELECTRIC (RIGHT) SELFSENSING ACTUATORS AT $346 \mathrm{~Hz}$ (TOP) AND $427 \mathrm{~Hz}$ (BOTTOM)

\section{CONCLUSION}

This paper addressed the problem of time-harmonic disturbance rejection using self-sensing actuators. Both the piezoceramic and electrodynamic transduction principles have been studied and implemented successfully. In both cases, an electromechanical model that fully encompasses the coupled plateactuator dynamic response is provided. Experimental results performed on a simply supported plate showed significant reduction of flexural energy using the two types of transducers. A common methodology is proposed, in which a flow variable proportional to the structure velocity is derived from electrical quantities, resulting in the use of a generic controller architecture. Further developments will now focus on ways to achieve narrow-band vibration reduction with the help of self-sensing actuators. In our forthcoming work, we are also interested in studying the performance of these self-sensing actuators in other control approaches such as active structural acoustic control.

\section{ACKNOWLEDGMENT}

This work was supported by the Swiss National Science Foundation, the National Sciences and Engineering Research Council of Canada, Bombardier Aerospace and the Fonds de Recherche Nature et Technologies du Québec. The authors wish to thank Mr. Denis Imbeault for his help in the design and assembly of electronic circuits. 


\section{REFERENCES}

[1] D. Guicking, J. Melcher and R. Wimmel, Active impedance control in mechanical structures, Acta Acustica united with Acustica, 69(2), 1989

[2] C. R. Fuller, S. J. Elliott and P. A. Nelson, Active control of vibration, Academic Press Inc., 1996

[3] C. K. Lee, Theory of laminated piezoelectric plates for the design of distributed sensors/actuators. Part I: Governing equations and reciprocal relationships, J. Acoust. Soc. Am., 87(3), 1990

[4] E. K. Dimitriadis, C. R. Fuller and C. A. Rogers, Piezoelectric actuator for distributed vibration excitation of thin plates, J. Vib. Acoust., 113(1), 1991

[5] S. J. Elliott, P. Gardonio, T. C. Sors and M. J. Brennan, Active vibroacoustic control with multiple local feedback loops, J. Acoust. Soc. Am., 111(2), 2002

[6] P. Gardonio and S. J. Elliott, Smart panels for active structural acoustic control, Smart Mater. Struc., 13, 2004

[7] Ch. Paulitsch, P. Gardonio and S. J. Elliott, Active vibration control using an inertial actuator with internal damping, J. Acoust. Soc. Am., 119(4), 2006

[8] S. J. Elliott, J. Rohlfing and P. Gardonio, Multifunctional design of inertially-actuated velocity feedback controllers, J. Acoust. Soc. Am., 131(2), 2012

[9] J. S. Vipperman and R. L. Clark, Implementation of an adaptive piezoelectric sensoriactuator, Am. Inst. of Aeronautics and Astronautics, 34(10), 1996

[10] S. J. Elliott, M. Serrand and P. Gardonio, Feedback stability limits for active isolation systems with reactive and inertial actuators, J. Vib. Acoust., 123(2), 2002

[11] N. W. Hagood and E. H. Anderson Simultaneous sensing and actuation using piezoelectric materials, Proc. SPIE 1543 Active and adaptive optical components, San Diego CA, 1991

[12] J. J. Dosch, D. J. Inman and E. Garcia, Self-sensing piezoelectric actuator for collocated control, J. of Intel. Mat. Syst. and Struct., 3(1), 1992.

[13] J. S. Vipperman and R. L. Clark, Multivariable feedback active structural acoustic control using adaptive piezoelectric sensoriactuators, J. Acoust. Soc. Am., 105(1), 1999

[14] S. O. R. Moheimani, A survey of recent innovations in vibration damping and control using shunted piezoelectric transducers, IEEE Trans. Control Syst. Tech., 11(4), 2003

[15] D.J. Leo and D. Limpert, A self-sensing technique for active acoustic attenuation, J. Sound and Vibration, 235(5), 2000

[16] B. Hanson and M. Levesley, Self-sensing applications for electromagnetic actuators, Sensors and Actuators A: Physical, 116, 2004

[17] F. Fahy and P. Gardonio, Sound and structural vibration : Radiation, transmission and response, Elesevier Academic Press, 2007

[18] IEEE Standard on Piezoelectricity, ANSI/IEEE, 1987
[19] M. Michau, A. Berry, P. Herzog and P. Micheau, Bending nearfield compensation in the context of vibroacoustic active control, Proceedings of the 21ième Congrès Français de Mécanique, Aug. 26-30, Bordeaux, France, 2013.

[20] M. Neubauer, A. Renner and J. Wallaschek, Model-based piezoelectric self-sensing technique, Proceedings of the ASME 10th Biennial Conf. on Eng. Syst. Design and Analysis, July 12-14, Istanbul, Turkey, 2010

[21] A. Pelletier, P. Micheau and A. Berry, Implementation of a self-sensing piezoelectric actuator for vibro-acoustic active control, Proceedings of the SPIE, 9061, San Diego, 2014

[22] M. J. Guan and W. H. Liao. On the equivalent circuit models of piezoelectric ceramics. Ferroelectrics, 386(1), 2009

[23] C. H. Hansen and S. D. Snyder, Active Control of Noise and Vibration, E FN Spon, 1997

[24] O. Delas, A. Berry, P. Masson and Y. Pasco, Optimizing the thickness of piezoceramic actuators for bending vibration of planar structures, J. of Intel. Mat. Syst. and Str., 18, 2007

[25] P. Micheau, R. Louviot and A. Berry, Decentralized resonant controller for vibroacoustic active control, Proceedings of the 15th Mediterranean Conference on Control and Automation, July 27-29, Athens, 2007

[26] P. Micheau and S. Renault, Active control of the complex envelope associated with a low damped mode, Mech. Syst. and Signal Proc., 20(3), 2006 